'llu. Revista de Ciencias de las Religiones

ISSN: 1135-4712

http://dx.doi.org/10.5209/ILUR.57427

\title{
Las tablillas micénicas de Tebas y la religión griega ${ }^{1}$
}

Juan Piquero Rodríguez²

[en] The Mycenaean Tablets from Thebes and the Greek Religion

Esquema:

1. Introducción

2. Ediciones

3. Los editores y la interpretación religiosa

4. La crítica y la interpretación laica

5. Valoraciones finales

\section{Introducción}

El 2 de noviembre de 1993, debido a una excavación de emergencia ocasionada por unas obras de canalización de la Odos Pelopidou, en la moderna ciudad de Tebas, salió a la luz un fragmento de tablilla en lineal B en formato página ${ }^{3}$. Vassilis. L. Aravantinos, el éforo a cargo del Servicio Arqueológico de Tebas en ese momento, ordenó suspender los trabajos de canalización de la calle y comenzó a trabajar en la excavación de la zona. Además, a unos $20 \mathrm{~m}$. al Este de donde se produjo el hallazgo se encuentra el llamado Arsenal, donde en 1964 aparecieron las primeras tablillas en lineal B de la ciudad, lo que llevó a Aravantinos a pensar que en esa zona podrían aparecer más documentos. Así, él y su equipo excavaron un terreno de unos $200 \mathrm{~m}^{2}$ durante unos quinientos días repartidos en dos campañas realizadas en los inviernos de 1993-1994 y 1994-19954.

Durante estas campañas arqueológicas se encontraron cerca de doscientas cincuenta tablillas en lineal $\mathrm{B}^{5}$. Para la edición de los textos hallados, Aravantinos contó con los profesores Louis Godart y Anna Sacconi, quienes en una serie de artículos y, sobre todo, en la esperada edición de los textos ofrecieron interpre-

Este trabajo se enmarca en el proyecto de investigación «Estudios de morfosintaxis nominal: lenguas paleohispánicas e indoeuropeas antiguas» (FFI2015-63981-C3-2). Agradezco su ayuda en la elaboración de este trabajo a Alberto Bernabé e Irene Serrano. Cualquier eventual error es solo responsabilidad del autor.

2 Universidad Complutense de Madrid (España).

E-mail: juanpiqu@ucm.es

3 Hoy conocida como TH Av 100

4 Sobre la excavación cf. Aravantinos - Godart - Sacconi 1995, 809-818; Serrano Laguna 2015, 24-26.

5 Cf. Serrano Laguna 2015, 42 sobre la cantidad exacta de las tablillas. 
taciones de índole religiosa para la mayor parte de los términos atestiguados en ellas. La crítica derivada de estas publicaciones generó una enorme polémica y ha desmontado prácticamente la totalidad de las hipótesis de los editores, estimando que, en general, todos aquellos términos considerados como designaciones divinas o vinculadas con la religión pueden ser tenidos verosímilmente por designaciones seculares.

Es precisamente la enorme cantidad de bibliografía generada en torno al posible contenido religioso o no de los documentos lo que ha hecho posible este boletín que pretende ser un instrumento de trabajo para aquellos que pretendan estudiar la problemática de las tablillas tebanas.

\section{Ediciones}

Antes del descubrimiento de las tablillas tebanas de la Odos Pelopidou, la edición de referencia para los escasos documentos que había arrojado el yacimiento era la de J. L. Melena - J.-P. Olivier (1991), TITHEMY. The Tablets and Nodules in Linear B from Tyrins, Thebes and Mycenae (Suplemento a Minos, 12), Ediciones de la Universidad de Salamanca - Servicio editorial de la Universidad del País Vasco.

El hallazgo de los documentos de la Odos Pelopidou, que fueron los que provocaron la polémica religiosa que nos ocupa, conllevó la aparición de nuevas ediciones.

V. L. Aravantinos - L. Godart-A. Sacconi (2001), Thèbes. Fouilles de la Cadmée I. Les tablettes en linéaire B de la Odos Pelopidou. Édition et commentaire, Pisa Roma, Istituti Editoriali e Poligrafici Internazionali.

Publicación de los documentos hallados en los años 90. Recoge las tablillas 100405. Introducción al contexto arqueológico y edición con fotos, facsímiles, transcripciones y comentario, en el que los editores desarrollan la teoría religiosa. Consta de índices, un glosario, concordancias y un signario.

V. L. Aravantinos - L. Godart - A. Sacconi (2002), Thèbes. Fouilles de la Cadmée III. Corpus des documents d'archives en linéare B de Thèbes (1-433), Pisa - Roma, Istituti Editoriali e Poligrafici Internazionali.

Edición de todos los textos tebanos ordenados por número y no por serie. Frente al volumen anterior, en el que solo están editadas las tablillas de la Odos Pelopidou, incluye todos los textos publicados previamente en TITHEMY más algunos fragmentos mínimos (406-420), los nódulos Wu 429-431 y la tablillas Up 432 y X 433. Consta de fotos, dibujos y transcripciones de las tablillas, una concordancia de los textos ordenados por números, un signario y un índice de palabras.

V. L. Aravantinos - M. Del Freo - L. Godart - A. Sacconi (2005), Thèbes. Fouilles de la Cadmée IV. Les textes de Thèbes (1-433). Translitération et tableaux des scribes, Pisa - Roma, Istituti Editoriali e Poligrafici Internazionali.

Esta es la edición de referencia para las inscripciones en lineal B de Tebas en la actualidad. Las novedades en relación con el volumen anterior son que los documentos están ordenados por series y que se incluye un amplio estudio paleográfico de las manos de los escribas. Consta además de concordancias por series, números, escribas y lugar de hallazgo, así como de signarios.

Con todo, para las fotos y los facsímiles debe recurrirse al volumen III y, para el comentario de los textos, al volumen I. 


\section{Los editores y la interpretación religiosa}

Aunque la edición definitiva de los textos se hizo esperar hasta 2001, los editores fueron publicando trabajos desde 1995 en adelante en los que afirmaban que la mayor parte de los textos de la Odos Pelopidou estaban relacionados con la religión, en particular con ofrendas de alimentos a divinidades, animales «sagrados» y sacerdotes que prestaban sus servicios en los santuarios de la ciudad.

Según ellos -y otros que les siguieron-, el término $m a-k a$ correspondería a $\mu \tilde{\alpha}$ Га̃ (A. Supp. 891 s.), una epíclesis de Deméter, la 'Madre Tierra'. Además, la diosa formaría una tríada con $k o-w a$, *кó $\rho F \alpha$, 'la Muchacha', esto es, Perséfone y con opo-re-i, dativo de *ỏ $\pi \omega \rho \varepsilon v ́ \varsigma$, una advocación de Zeus 'protector de los frutos'. Otros nuevos términos hacían también referencia a divinidades, como a-pu-wa, "A $\rho \pi v i \alpha$, la

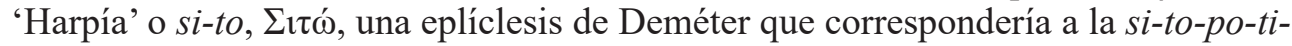
$n i-j a$ atestiguada en los documentos de Micenas (MY Oi 701).

En su opinión, la mayor parte de los apelativos que figuran en las tablillas harían referencia a desservants de sanctuaire. Así, a-ke-ne-u-si, a-ko-ro-da-mo / a-ko-damo, de-qo-no, e-pi-qo-i, e-re-u-te-ri, i-qo-po-qo, ka-ne-jo, ka-wi-jo, ku-na-ki-si, pere-wi-jo, si-to-ko-wo y to-pa-po-ro-i.

Además, en las tablillas habría referencias a fiestas y, en algunos casos, rituales mistéricos semejantes a los que tenían lugar en el primer milenio a.C. en Eleusis.

Trabajos que defienden la totalidad o la mayoría de estas interpretaciones son, en orden cronológico, los siguientes:

V. L. Aravantinos - L. Godart - A. Sacconi (1995), «Sui nuovi testi del palazzo di Cadmo a Tebe: note preliminari», $R A L$ 6/4, 809-844.

L. Godart -A. Sacconi (1996a), «Les dieux thébains dans les archives mycéniennes», CRAI 140 (1), 99-113.

L. Godart - A. Sacconi (1996b), «La triade tebana nei documenti in lineare B del palazzo di Cadmo», RAL 7/9, 283-285.

L. Godart - A. Sacconi (1997), «Les archives de Thèbes et le monde mycénien», CRAI 141 (3), 889-906.

M. Lejeune (1997a), «Anatomie de la série thébaine Gp», Mémoires de Philologie Mycénienne $4^{\text {ème }}$ Série (1969-1996), Roma, Incunabula Graeca, 279-281.

M. Lejeune (1997b), «Sur les offrandes thébains à Mère Terre», Mémoires de Philologie Mycénienne $4^{e ̀ m e}$ Série (1969-1996), Roma, Incunabula Graeca, 279-281.

A. Sacconi (1998), «I nuovi testi tebani ed il significato del termine ka-pa», RCCM 40, 281-288.

V. L. Aravantinos (1999), «Mycenaean Texts and Contexts at Thebes. The Discovery of New Linear B Archives on the Kadmeia», en S. Deger-Jalkotzy - S. Hiller - O. Panagl (eds.), Floreant Studia Mycenaea. Akten des X Internationalen Mykenologischen Colloquiums in Salzburg vom 1.-5. Mai 1995, Wien, Verlag der Österreichischen Akademie der Wissenschaften, 45-78.

M. Del Freo (1999), «Mic. ke-re-na-i nei nuovi testi in lineare B di Tebe», en V. La

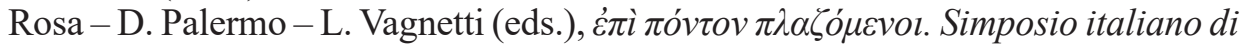
Studi Egei dedicato a Luigi Bernabò Brea e Giovanni Pugliese Carratelli. Roma, 18-20 febbraio 1998, Roma, Scuola archeologica italiana di Atene, 299-304.

L. Godart - A. Sacconi (2000), «Tebe, Demetra ed Eleusi», en P. A. Bernardini (ed.), Presenza e funzione della città di Tebe nella cultura greca: Atti del Convegno 
Internazionale (Urbino 7-9 Iuglio 1997), Pisa - Roma, Istituti Editoriali e Poligrafici Internazionali, 17-26.

A. Sacconi (2000), «Continuità di culti in Beozia. Lo Zeus opores di Akraiphia», en

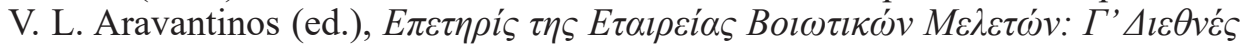

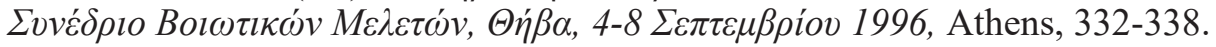

M. Del Freo (2001), reseña a V. L. Aravantinos - L. Godart - A. Sacconi (2001), Thèbes. Fouilles de la Cadmée I. Les tablettes en linéaire B de la Odos Pelopidou. Édition et commentaire, Pisa - Roma, Istituti Editoriali e Poligrafici Internazionali, RFIC 129, 78-91.

L. Godart (2001), «La Terre Mère et le monde égéen», en R. Laffineur - R. Hägg (eds.), POTNIA. Deities and Religion in the Aegean Bronze Age. Proceedings of the $8^{\text {th }}$ International Aegean Conference Göteborg, Göteborg University, 12-15 April 2000 [Aegaeum 22], Liège: Université de Liège; Austin: University of Texas at Austin, Peeters, 463-466.

D. Rousioti (2001), «Did the Mycenaeans Believe in Theriomorphic Divinities?», en R. Laffineur - R. Hägg (eds.), POTNIA. Deities and Religion in the Aegean Bronze Age. Proceedings of the $8^{\text {th }}$ International Aegean Conference Göteborg, Göteborg University, 12-15 April 2000 [Aegaeum 22], Liège: Université de Liège; Austin: University of Texas at Austin, Peeters, 305-314.

A. Sacconi (2001), «Les repas sacrés dans les textes mycéniens», en R. Laffineur - R. Hägg (eds.), POTNIA. Deities and Religion in the Aegean Bronze Age. Proceedings of the $8^{\text {th }}$ International Aegean Conference Göteborg, Göteborg University, 12-15 April 2000 [Aegaeum 22], Liège: Université de Liège; Austin: University of Texas at Austin, Peeters, 467-470.

V. L. Aravantinos - L. Godart-A. Sacconi (2003), «En marge des nouvelles tablettes en linéaire B de Thèbes», Kadmos 42, 15-30.

C. Milani (2003), «La Madre Terra nei nuovi testi micenei di Tebe», Aevum 77, 3-8.

C. J. Ruijgh (2003), reseña a V. L. Aravantinos - L. Godart - A. Sacconi (2001), Thèbes. Fouilles de la Cadmée I. Les tablettes en linéaire B de la Odos Pelopidou. Édition et commentaire, Pisa - Roma, Istituti Editoriali e Poligrafici Internazionali, Mnemosyne 56, 219-228.

C. Milani (2004), «Destinatari di orzo nei nuove tavolette micenee di Tebe: il caso di Fq 254», en G. Rocca (ed.), Dialetti, dialettismi, generi letterari e funzioni sociali. Atti del V Colloquio Internazionale di Linguistica Greca, Milano 12-13 settembre 2002, Alessandria, Edizioni dell'Orso, 357-366.

C. J. Ruijgh (2004), «La Mère Terre dans les textes grecs classiques», en B. Gentili L. Godart - C. Prato (eds.), I culti primordiali della grecità alla luce delle scoperte di Tebe. Convegno internazionale, Roma, 24-25 febbraio 2000, Roma, Academia Nazionale dei Lincei, 81-100.

A. Sacconi (2004), «I testi in lineare B della Odos Pelopidou: la finalità amministrativa ed il contesto cultuale», en B. Gentili - L. Godart - C. Prato (eds.), I culti primordiali della grecità alla luce delle scoperte di Tebe. Convegno internazionale, Roma, 24-25 febbraio 2000, Roma, Academia Nazionale dei Lincei, $31-46$.

C. J. Ruijgh (2004), «À propos des nouvelles tablettes de Thèbes, I. Les trois divinités $m a-k a, o-p o-r e-i$ et $k o-w a$ et les trois subordonnées temporelles dans la série Fq», Mnemosyne 57, 1-44.

M. Iodice (2005), «Nomi di animali nei testi micenei di Tebe», Aevum 79, 9-16. 
C. Milani (2005), «Le nuove tavolette di Tebe: note su Lacedemoni e Tebani», Aevum 79, 3-7.

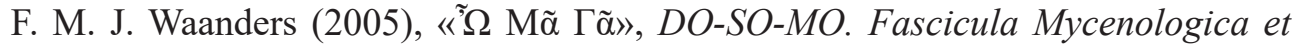
Classica Polona 7, 117-121.

C. Milani (2006), «De Tebe a Pilo: processi scribali nei documenti micenei», Aevum $80,5-12$.

C. J. Ruijgh (2006), «The Three Temporal Clauses (TH Fq 126; 130; 254)», en S. Deger-Jalkotzy - O. Panagl (eds.), Die neuen Linear B-Texte aus Theben. Ihr Aufschlußwert für die mykenische Sprache und Kultur. Akten des internationalen Forschungskolloquiums an der Österreichischen Akademie der Wissenschaften. 5-6 Dezember 2002, Wien, Verlag der Österreichischen Akademie der Wissenschaften, 159-170.

M. Iodice (2008), «Miceneo $a-p u-w a »$, en A. Sacconi - M. Del Freo - L. Godart M. Negri (eds.), Colloquium Romanum. Atti del XII Colloquio Internazionale di Micenologia. Roma, 20-25 febbraio 2006 (Pasiphae 2), Pisa - Roma, Fabrizio Serra Editore, 361-368.

C. Milani (2010), «Scribi tebani: il caso delle mani 307, 308, 309», Aevum 84, 1-5.

K. T. Witczak (2011), «Dioscuri in the Mycenaean Times», DO-SO-MO. Fascicula Mycenologica et Classica Polona 9, 30-41.

F. M. J. Waanders (2012), «Who is who in the Mycenaean Zoo?», en P. Hr. Ilievski V. Mitevski - R. Duev (eds.), Ad perpetuam memoriam Michaelis D. Petruševski. Proceedings of the International Conference. 100th Anniversary of the Birth of Professor Mihail D. Petruševski (1911-1990), ZAnt. Monographs 10, 295-303.

N. Manzano (2013-2014), Le triadi divine nei testi micenei: elementi di rottura e continuità con $i$ datti del primo millenio. Tesis doctoral leída en la Facoltà de Lettere e Filosofia de La Sapienza (http://padis.uniroma1.it/handle/10805/2512).

A. Franceschetti (2016), «La religione micenea», en M. Del Freo - M. Perna, Manuale di epigrafia micenea. Introduzione allo studio dei testi in lineare $B$, vol. II, Limena PD, Libreriauniversitaria.it edizioni, 725-751.

N. Manzano (2016), «Miceneo si-to», Pasiphae 10, 91-96.

M. Tozza (2016), Animales y dioses en la Grecia prehomérica, Zaragoza, Prensas universitarias de Zaragoza.

\section{La crítica y la interpretación laica}

Las críticas a toda esta interpretación religiosa de las tabillas no se hicieron esperar. Desde temprano se puso de manifiesto la poca verosimilitud de la tríada divina $m a-k a$, $o-p o-r e-i, k o-w a$ y, con esto, la totalidad del sistema religioso eleusino-tebano, con divinidades como a-pu-wa o si-to. Esta negativa a aceptar la interpretación religiosa condicionó también el análisis propuesto en relación con los apelativos de persona y con las menciones de animales. Según la opinión más aceptada, los apelativos harían referencia a grupos de personas seculares que desempeñan funciones en ceremonias religiosas, aunque se han propuesto otras hipótesis. En este mismo sentido, las referencias a animales designarían verosímilmente a grupos de personas involucradas en el culto que portarían máscaras de animales y participarían en una ceremonia religiosa. Pese a que esta parece la teoría más verosímil, se han propuesto también otras opciones. 
La siguiente bibliografía reúne la mayor parte de las críticas emitidas contra la hipótesis religiosa de los editores:

J. Chadwick (1996-1997 [1998]), «Three Temporal Clauses», Minos 31-32, 293-301.

J. T. Killen (1999), «Some observations on the New Thebes Tablets», BICS 43, $217-$ 219.

J. L. Melena (2001), Textos griegos micénicos comentados, Vitoria, Parlamento Vasco.

J.-L. Perpillou (2001), «Les nouvelles tablettes de Thèbes (Autour d'une publication récente)», reseña a V. L. Aravantinos - L. Godart - A. Sacconi (2001), Thèbes. Fouilles de la Cadmée I. Les tablettes en linéaire B de la Odos Pelopidou. Édition et commentaire, Pisa - Roma, Istituti Editoriali e Poligrafici Internazionali, $R P h$ 75, 307-315.

Th. G. Palaima (2000-2001[2002]), reseña a V. L. Aravantinos - L. Godart - A. Sacconi (2001), Thèbes. Fouilles de la Cadmée I. Les tablettes en linéaire B de la Odos Pelopidou. Édition et commentaire, Pisa - Roma, Istituti Editoriali e Poligrafici Internazionali, Minos 35-36, 475-486.

F. Rougemont (2001 [2003]), reseña a V. L. Aravantinos - L. Godart - A. Sacconi (2001), Thèbes. Fouilles de la Cadmée I. Les tablettes en linéaire B de la Odos Pelopidou. Édition et commentaire, Pisa - Roma, Istituti Editoriali e Poligrafici Internazionali, y a V. L. Aravantinos - L. Godart - A. Sacconi (2002), Thèbes. Fouilles de la Cadmée III. Corpus des documents d'archives en linéare B de Thèbes (1-433), Pisa - Roma, Istituti Editoriali e Poligrafici Internazionali, Topoi 11, 689-706.

Y. Duhoux (2002-2003 [2007]), «Dieux ou humains? Qui sont ma-ka, o-po-re-i et ko-wa dans le tablettes linéaire B de Thèbes?, Minos 37-38, 173-253.

S. James (2002-2003 [2007]), «The Thebes Tablets and the Fq series. A contextual analysis», Minos 37-38, 397-417.

N. Guilleux (2003), reseña a V. L. Aravantinos - L. Godart - A. Sacconi (2001), Thèbes. Fouilles de la Cadmée I. Les tablettes en linéaire B de la Odos Pelopidou. Édition et commentaire, Pisa - Roma, Istituti Editoriali e Poligrafici Internazionali, BSL 98, 262-268.

Th. G. Palaima (2003a), reseña a V. L. Aravantinos - L. Godart - A. Sacconi (2001), Thèbes. Fouilles de la Cadmée I. Les tablettes en linéaire B de la Odos Pelopidou. Édition et commentaire, Pisa - Roma, Istituti Editoriali e Poligrafici Internazionali, AJA 107, 113-115.

Th. G. Palaima (2003b), «Reviewing the New Linear B Tablets from Thebes», Kadmos 42, 31-38.

C. Boëlle (2004), Po-ti-ni-ja: l'element féminin dans la religion mycénienne (d'après les archives en linéaire B), Nancy, A.D.R.A.

M. L. Nosch (2004), reseña a V. L. Aravantinos - L. Godart - A. Sacconi (2001), Thèbes. Fouilles de la Cadmée I. Les tablettes en linéaire B de la Odos Pelopidou. Édition et commentaire, Pisa - Roma, Istituti Editoriali e Poligrafici Internazionali, Gnomon 76, 535-541.

C. Trümpy (2004), «Die Thesmophoria, Brimo, Deo und das Anaktoron. Beobachtungen zur Vorgeschichte des Demeterkults», Kernos 17, 13-42.

A. Uchitel (2004), «Theban Fq series and Ancient Near Eastern Messenger's Stations», SMEA 46, 271-279.

Y. Duhoux (2005), «Les nouvelles tablettes en linéaire B de Thèbes et la religion grecque», $A C 74,1-19$. 
Y. Duhoux (2006a), «Adieu au $m a-k a$ cnossien. Une nouvelle lecture on KN F 51 et ses conséquences pour les tablettes linéaire B de Thèbes», Kadmos 45, 1-19.

Y. Duhoux (2006b), «La soi-dissant "triade divine" des tablettes linéaire B de la rue Pélopidou (Thèbes)», en M. R. Cataudella - A. Greco - G. Mariotta (eds.), Gli storici e la lineare B cinquant'anni dopo. Atti del convegno internazionale. Firenze, 24-25 Novembre 2003 (Acta Sileni 1), Padova, S.A.R.G.O.N, 65-82.

J. L. García Ramón (2006), «Zu den Personennamen der neuen Texte aus Theben», en S. Deger-Jalkotzy - O. Panagl (eds.), Die neuen Linear B-Texte aus Theben. Ihr Aufschlußwert für die mykenische Sprache und Kultur. Akten des internationalen Forschungskolloquiums an der Österreichischen Akademie der Wissenschaften. 5-6 Dezember 2002, Wien, Verlag der Österreichischen Akademie der Wissenschaften, 37-52.

J.T. Killen (2006), «Thoughts on the functions of the new Thebes tablets», en S. Deger-Jalkotzy - O. Panagl (eds.), Die neuen Linear B-Texte aus Theben. Ihr Aufschlußwert für die mykenische Sprache und Kultur. Akten des internationalen Forschungskolloquiums an der Österreichischen Akademie der Wissenschaften. 5-6 Dezember 2002, Wien, Verlag der Österreichischen Akademie der Wissenschaften, 79-110.

M. Meier-Brügger (2006), «Sprachliche Beobachtungen», en S. Deger-Jalkotzy - O. Panagl (eds.), Die neuen Linear B-Texte aus Theben. Ihr Aufschlußwert für die mykenische Sprache und Kultur. Akten des internationalen Forschungskolloquiums an der Österreichischen Akademie der Wissenschaften. 5-6 Dezember 2002, Wien, Verlag der Österreichischen Akademie der Wissenschaften, 111-118.

G. Neumann (2006), «...Gans und Hund und ihresgleichen...», en S. Deger-Jalkotzy - O. Panagl (eds.), Die neuen Linear B-Texte aus Theben. Ihr Aufschlußwert für die mykenische Sprache und Kultur. Akten des internationalen Forschungskolloquiums an der Österreichischen Akademie der Wissenschaften. 5-6 Dezember 2002, Wien, Verlag der Österreichischen Akademie der Wissenschaften, 125-138.

Th. G. Palaima (2006), «*65 = FAR? Or $j u$ ? and other interpretative conundra in the new Thebes tablets», en en S. Deger-Jalkotzy - O. Panagl (eds.), Die neuen Linear B-Texte aus Theben. Ihr Aufschlußwert für die mykenische Sprache und Kultur. Akten des internationalen Forschungskolloquiums an der Österreichischen Akademie der Wissenschaften. 5-6 Dezember 2002, Wien, Verlag der Österreichischen Akademie der Wissenschaften, 139-158.

G. Ricciardelli (2006), «I nomi di animali nelle tavolette di Tebe: una nouva ipotesi», $P P$ 61, 241-263.

Y. Duhoux (2007), «Le nom du 'fils' en linéaire B», en F. Lang - C. Reinholdt

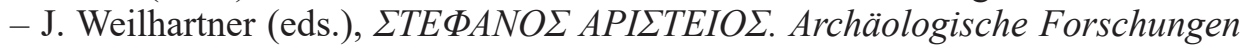
zwischen Nil und Istros. Festschrift für Stefan Hiller zum 65. Geburtstag, Wien, Phoibos Verlag, 95-103.

J. Weilhartner (2007), «Die Tierbezeichnungen auf den neuen Linear B-Texten aus Theben», en E. Alram-Stern - G. Nightingale (eds.), Keimelion: Elitenbildung und elitärer Konsum von der mykenischen Palastzeit bis zur homerischen Epoche, Akten des internationale Kongresses vom 3. bis 5. Februar in Salzburg (ÖAW, Philosophisch-historische Klasse, Denkschriften 350), Wien, Verlag der Österreichischen Akademie der Wissenschaften, 339-351.

A. Bernabé (2008), «Tres tablillas micénicas de Tebas (TH Av 100, 101 y 104)», en A. Alemany (ed.), $\dot{\alpha} \kappa \rho i ́ \beta \varepsilon \imath \alpha \tau \tilde{\omega} v \lambda \varepsilon \chi \theta \dot{v} v \tau \omega v$. Homenatge a Rosa-Araceli Santiago Álvarez (Faventia 30), 17-31. 
Y. Duhoux (2008a), «Animaux ou humains? Réflexions sur les tablettes Aravantinos de Thèbes, en A. Sacconi-M. Del Freo - L. Godart-M. Negri (ed.), Colloquium Romanum». Atti del XII Colloquio internazionale di micenologia, Roma 20-25 febbraio 2006 (Pasiphae 2), Pisa - Roma, Fabrizio Serra Editore, 231-250.

Y. Duhoux (2008b), «Mycenaean Anthology», en Y. Duhoux - A. Morpurgo (eds.), A Companion to Linear B: Mycenaean Greek Texts and their World, vol. 1, Louvain-la-Neuve - Dudley, MA, 243-393.

Th. G. Palaima (2008), «The significance of Mycenaean Words relating to meals, meal rituals, and food», en L. Hitchcock - R. Laffineur - J.L. Crowley (eds.), DAIS. The Aegean Feast, Proceedings of the 12th International Aegean Conference, University of Melbourne, Centre for Classics and Archaeology, 25-29 March 2008 (Aegaeum 29), Liège - Austin, Peeters, 383-389.

R. Palmer (2008), "Wheat and Barley in Mycenaean Society 15 Years Later», en A. Sacconi - M. Del Freo - L. Godart - M. Negri (ed.), Colloquium Romanum. Atti del XII Colloquio internazionale di micenologia, Roma 20-25 febbraio 2006 (Pasiphae 2), Pisa - Roma, Fabrizio Serra Editore, 621-640.

F. Rougemont (2009), «Comment interpréter les mentions d'animaux dans les nouveaux textes mycéniens de Thèbes?, en N. Guilleux - D. Petit (eds.), A-TIDO-RO. Etrennes en hommage à Jean-Louis Perpillou de la part de quelquesuns de ses anciens élèves (Ktema 34), 103-126.

C. Boëlle (2010), «po-ti-ni-ja ... dans tous ses états», en I. Boehm - S. Müller Celka (eds.), Espace civil, espace religieux en Égée durant la période mycénienne (TMO 54), Lyon, Maison de l'Orient et de la Méditerranée, 35-50.

J. L. García Ramón (2010), «Espace religieux, théonymes, épiclèses. À propos des nouveaux textes thébains», en I. Boehm - S. Müller Celka (eds.), Espace civil, espace religieux en Égée durant la période mycénienne (TMO 54), Lyon, Maison de l'Orient et de la Méditerranée, 73-92.

N. Guilleux (2010), «La religion dans les nouvelles tablettes de Thèbes. Réflexions complémentaires», en I. Boehm - S. Müller Celka (eds.), Espace civil, espace religieux en Égée durant la période mycénienne (TMO 54), Lyon, Maison de l'Orient et de la Méditerranée, 93-102.

A. Bernabé (2011), «Las tablillas Fq 126 y 130 de Tebas», en M. J. García Blanco T. Amado Rodríguez - M. J. Martín Velasco - A. Pereiro Pardo - M. E. Vázquez

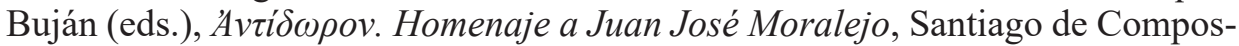
tela, Universidade de Santiago de Compostela, 57-70.

A. Bernabé - I. Serrano (2011), «Nuevos datos sobre la religión de la Tebas micénica: las tablillas de la Odos Pelopidou», en E. Calderon Dorda - A. Morales Ortiz (eds.), EUSÉBEIA. Estudios de religión griega, Madrid, Signifer Libros, 11-35.

S. Hiller (2011), «Mycenaean Religion and Cult», en Y. Duhoux -A. Morpurgo (eds.), A Companion to Linear B: Mycenaean Greek Texts and their World, vol. 2, Louvain-la-Neuve - Walpole, MA, Peeters, 169-212.

B. Montecchi (2011), «The Allotments of HORD and VIN to carpenters (te-ka-ta-si) at Thebes (TH Fq 247, Gp 112, 114, 147, 175), SMEA 53, 171-187.

A. Bernabé (2012a), «Posibles menciones religiosas en las tablillas de Tebas», en C. Varias García (ed.), Actas del simposio internacional: 55 años de micenología (1952-2007) (Faventia Supplementa 1), 183-206.

A. Bernabé (2012b), «TH Av 101 and Mycenaean to-pa-po-ro(-i)», en P. Carlier Ch. De Lamberterie - M. Egetmeyer - N. Guilleux - F. Rougemont - J. Zurbach 
(eds.), Études mycéniennes 2010. Actes du XIIIe colloque international sur les textes égéens, Sèvres, Paris, Nanterre 20-23 septembre 2010 (Pasiphae 10), Pisa - Roma, Fabrizio Serra Editore, 167-176.

J. L. García Ramón (2012), «En travaillant à une grammaire du mycénien. 1. a-pie-qe lamp ${ }^{h} i^{h} e n k^{w} e /$ «(on) mentionna, (on) énumera». 2. Absence d'augment et mode injonctif. 3. di-ri-mi-jo: Drimios, fils de Zeus», en P. Carlier - Ch. De Lamberterie - M. Egetmeyer - N. Guilleux - F. Rougemont - J. Zurbach (eds.), Études mycéniennes 2010. Actes du XIIIe colloque international sur les textes égéens, Sèvres, Paris, Nanterre 20-23 septembre 2010 (Pasiphae 10), Pisa Roma, Fabrizio Serra Editore, 436-454.

I. Serrano Laguna (2012), «Una nueva interpretación del término micénico e-pe-toi», en A. Cabedo Nebot - P. Infante Ríos (eds.), Lingüistica XL. El lingüísta del siglo XXI, Madrid, SEL Ediciones, 241-246.

M. Del Freo (2014 [2015]), «The allocations of HORD to $m a-k a$ and de-qo-no in the Fq tablets from Thebes», Kadmos 53, 71-78.

J. L. García Ramón (2014), «Two personal names (Dative me-to-re-i and o-po-rei) and a Place Name (Directive me-to-re-ja-de) in the Mycenaean Thebes», en D. Nakassis - J. Gulizio - S.A. James (eds.), KE-RA-ME-JA: Studies Presented to Cynthia W. Shelmerdine (Prehistory Monographs 46), Philadelphia, INSTAP Academic Press, 283-292.

I. Serrano Laguna (2014), «Danzas de animales micénicas», en A. Bernabé - E. R. Luján (eds.), Donum Mycenologicum. Mycenaean Studies in Honour to Francisco Aura Jorro, Louvain-la-Neuve - Walpole, MA, Peeters, 163-172.

C. Varias García (2014), «"Snakes" in the Mycenaean Texts? On the interpretation of the Linear B term e-pe-to-i», en D. Nakassis - J. Gulizio - S. A. James (eds.), KE-RA-ME-JA: Studies Presented to Cynthia W. Shelmerdine (Prehistory Monographs 46), Philadelphia, INSTAP Academic Press, 179-190.

J. Henry (2015), «Le terme to-pa-po-ro-i en mycénien. Un état de la question», Res Antiquae 12, 95-102.

I. Serrano Laguna (2015), La religión micénica en las tablillas de Tebas. Tesis doctoral leída en la Facultad de Filología de la Universidad Complutense de Madrid (http://eprints.ucm.es/39765/).

J. L. García Ramón (2017), «Las instituciones micénicas a la luz de los textos en Lineal B: interpretaciones globales, filología y gramática del griego», en M. Oller - J. Pàmias - C. Varias (eds.). Tierra, territorio y población en la Grecia antigua: aspectos institucionales y míticos, Mering, Utopica, 81-110.

\section{Valoraciones finales}

La interpretación religiosa de los editores cada vez pierde más adeptos. Dada la enorme polémica generada en torno a los documentos tebanos de la Odos Pelopidou, no es fácil sacar conclusiones infalibles sobre su contenido. Con todo, como ya propusiera Th. G. Palaima (2000-2001 [2002]), (2008) y tal y como últimamente han defendido M. Del Freo (2014 [2015]) e I. Serrano (2015) probablemente la clave de la interpretación de las tablillas está en que $m a-k a$ no es un receptor, sino un término que designaría un tipo de HORD o similar. Si esto es así, la pretendida tríada tebana no habría existido nunca. KN F 51 apunta también en esta misma dirección. 
Por lo que respecta a los apelativos de persona y a las raciones de comida que estos reciben, se trata verosímilmente de festival rations (Killen 2006) que se otorgan a personas seculares que desempeñan funciones en ceremonias religiosas. Los nombres de animales, por su parte, designarían probablemente a personas que participarían en el ritual vestidas con pieles de animales y caracterizadas con máscaras y, tal vez, efectuando algun tipo de danza. En este sentido, véanse los trabajos de G. Ricciardelli (2006), J. Weilhartner (2007) e I. Serrano (2014) y (2015).

Para una actualización y evalucación crítica de la religión micénica en las tablillas tebanas, véase la tesis doctoral de I. Serrano (2015). 